\title{
COMPARATIVE ANALYSIS OF THE EXPERIENCE OF LONELINESS BETWEEN BULGARIANS AND RUSSIANS
}

\author{
Nataliya Alexandrova ${ }^{1}$, Liliya Babakova ${ }^{2}$, Inna Murtazina ${ }^{3}$, Olga Strizhitskaya $^{4}$, \\ Marina Petrash, ${ }^{5}$ Yuliya Chankova ${ }^{6}$
}

\begin{abstract}
Loneliness is a painful and alienating experience, which is an increasingly widespread phenomenon all over the globe. However, this phenomenon is perceived and interpreted differently in the world. The present study aims to compare the emotional, social, and romantic loneliness between Bulgarians and Russians. The two Slavic countries are historically and culturally close, but at the same time, there are many differences. Some studies have found that in the minds of both Russian and Bulgarian young people, loneliness is associated with both negative and positive aspects. This creates a common ground for the analysis and interpretation of this phenomenon in both countries. But the Russian people are more characterized by a positive-resource view of loneliness, while Bulgarian young people emphasize the negative. The study involved 608 people from Bulgaria and Russia aged 18-73 years. They completed the short version of the Social and Emotional Loneliness Scale for Adults by Di Tommaso E., Brannen C., Best L.A. (2004). The results indicated that ,romantic loneliness"was108 the most pronounced and present with its frequency in the content of the overall experience of loneliness. This outlines deficits, expectations, and emotions that are realized / function / in the deeply personal world of the person, of the studied groups of adults both in Bulgaria and in Russia. In second place in terms of its representation is the "social loneliness", which stands out among the studied adults from Bulgaria according to its marginal values. In the third place as third structural-content component is the "emotional loneliness", which is especially present in the experiences of the respondents from Bulgaria in comparison with those from Russia. Statistically significant results on the factors "country", "age" and "marital status" are also discussed.
\end{abstract}

UDC Classification: 005, 378, DOI: https://doi.org/10.12955/pss.v2.194

Keywords: loneliness, differences, Bulgarian, Russian example

\section{Introduction}

Loneliness is defined as a subjective, unpleasant, and stressful experience that results from the difference between the desired and achieved quality in a social group and is problematic for human mental health (Vanhalst et al., 2014; Zhao et al., 2018). There are two approaches to studying loneliness: One approach is a one-dimensional approach that views loneliness as a global phenomenon and measures loneliness as a single and holistic outcome (Russell, 1982). Other researchers suggest a multidimensional approach. Weiss (1973) and Junttila et al. (2013) offer two main dimensions of loneliness - social and emotional loneliness. In social loneliness, the individual experiences a lack and dissatisfaction with his social network of friends and acquaintances. Emotional loneliness is due to a lack of close relationships and attachment. DiTomasso and Spinner (1993) subsequently proposed a new tripartite theory of loneliness, based on which a scale is made, which assesses solitude in three aspects: social, emotional, and intimatepersonal. In this approach, loneliness, experience with the partner and in the family is considered part of emotional loneliness. The distinction between these three types of loneliness shows that people can be lonely in one domain and be satisfied in another domain (DiTommaso \& Spinner, 1993). For example, a person may be happy with his social network of friends and at the same time be in love alone. The aim of this study is to compare the levels of loneliness (emotional, social, and romantic/ intimatepersonal) between Bulgarians and Russians in adulthood. Bulgarians and Russians speak Slavic and lexically related languages. At the same time, these countries differ in size, resources, and traditions, suggesting differences in psychological and social characteristics (Strizhitskaya et al., 2020).

\section{Literature review}

Relationships with others provide us with a variety of social components such as attachment and social integration. Some research shows that married people and people living on a family basis are less single than unmarried, divorced, and widowed people. Given this, a lack of close relationships and attachment leads to emotional loneliness (Weiss, 1973), while a lasting partnership prevents such experiences. In addition, the death of the partner is associated with greater loneliness, which is more pronounced in

\footnotetext{
${ }^{1}$ International Business School, Sofia, Bulgaria, alexandrovan@yahoo.com

${ }^{2}$ Academy of Music, Dance and Fine Arts „Prof.Asen Diamandiev“, Faculy of Music Education, Plovdiv, Bulgaria, babakova_lilia@abv.bg

${ }^{3}$ Saint Petersburg State University, Saint Petersburg, Russian Federation, murtinna@yandex.ru

${ }^{4}$ Saint Petersburg State University, Saint Petersburg, Russian Federation, strizhitskaya@mail.ru

${ }^{5}$ Saint Petersburg State University, Saint Petersburg, Russian Federation, m.petrash@ spbu.ru

${ }^{6}$ Plovdiv University "Paisii Hilendarski”, Plovdiv, Bulgaria, j_chankova@abv.bg
} 
men, as the wife is the one who preserves and protects family relationships and preserves traditions (Lacković-Grgin, 2008).

The present study examines the problem of cross-cultural differences in perceptions of loneliness. Russia and Bulgaria are historically and culturally two close countries with Slavic roots and similar languages. At the same time, they differ in size, resources, and traditions, leading to differences in psychological characteristics.

There are some Russian studies on loneliness that view it mostly in a negative light. For instance, the Russian researcher M. Safonova (2012) studies the problem of loneliness in a sample of 120 Russians and found that the social ideas of modern Russians about the image of the lonely person are very diverse. According to her, Russians' social perceptions of the lonely person are very similar to the notions of a depressed person. After further research, she identified three prototypes for a lonely man: "careerist", "lone wolf" and "homemaker". In the minds of Russians, the lonely person has a low social status retired, disabled, people of other nationalities, poor people, single mothers, divorced, i.e., the lonely person does not meet the high standards of social success. There are also negative attitudes and negative stereotyping about the "typical" behavior of the old man, namely that the experience of loneliness is usually his fault (Safonova, 2012). Other Russian authors also view loneliness positively and even introduce the term "positive loneliness," which promotes personal growth (Osin\&Leontiev, 2013).

A study conducted among 108 Bulgarian respondents found that some economic factors had an impact and were predictors of the experience of loneliness (Alexandrova et al., 2020). Income, late payments of bills and loans, marital status can be significant factors in experiencing loneliness, which can affect the overall satisfaction and well-being of a man. Moreover, they offer a new approach to defining loneliness as a norm, pre-pathological and pathological. The criteria that a person has in relation to their relationships are also important because they are socially isolated but do not feel lonely. Some people have a lot of social contacts and still feel lonely. i.e., people perceive loneliness differently. The experience of the person and the crises he has gone through are also important. Some do not experience loneliness at all or experience it to a small degree. Usually, these people have felt the need for loneliness in previous stages of their lives (Alexandrova et al., 2020).

In a comparative study of a sample of Bulgarian and Russian young people with the help of content analysis, it was found that in both groups, loneliness was associated with mostly negative aspects. Still, at the same time, it may have some positive aspects (rest, solitude). Also, the results of the study showed that regardless of whether loneliness is associated with positive or negative aspects, in general, both Bulgarians and Russians perceive it in a similar way (Strizhitskaya et al.,2020). The Russian sample turned out to have a more positive view of loneliness, while Bulgarians tended to have a negative connotation. In general, Bulgarian society is more oriented towards traditional values, and one of these values is inclusion in society and the family system. Therefore, the fear of remaining in isolation is greater. Such assumptions are difficult to make for a Russian society, as they are characterized by greater variability in cultural, ethnic, and religious attitudes. But in general, it can be argued that both Bulgarians and Russians have a common field of meaning for the concept of "loneliness", which is not surprising given the historical closeness and friendship between Russians and Bulgarians (Strizhitskaya et al., 2020).

\section{Data and methodology}

The aim of the present study is to compare the levels of loneliness (emotional, social, and intimatepersonal) between Bulgarians and Russians in adulthood.

Subjects. The study involved 608 people, 290 from Bulgaria and 318 from Russia. $36 \%$ of the respondents were men, $63 \%$ - women. $13 \%$ were unmarried, $7 \%$ were single, $52 \%$ were married, $15 \%$ were divorced, and $11 \%$ were widowed. Of all subjects, $25.8 \%$ were in early adulthood (20-39 years), $45.1 \%$ were in middle adulthood (40-60 years), the remaining $29 \%$ were in late adulthood (over 60 years). By education factor, the sample consisted of $10 \%$ with primary education, $22 \%$ with secondary education, $9.1 \%$ with a bachelor's degree, $40.9 \%$ with a master's degree, and $17 \%$ indicated that they had different levels of education.

Instruments:

Demographic questionnaire - gender, age, education, marital status 
A Short Version of the Social and Emotional Loneliness Scale for Adults by Di Tommaso E., Brannen C., Best L.A. (2004). The scale was adapted and validated by N. Alexandrova (2015) for the Bulgarian sample, and for the Russian sample, it was adapted by Strizhitskaya et al. (2020). The questionnaire includes 15 items, which are formed in three scales:

1. A scale for emotional loneliness, which assesses the experience of loneliness within the family, the lack of understanding and support.

2. Social loneliness scale, which describes the state in which a person feels lonely within his social group.

3. Scale of romantic/intimate-personal loneliness, which examines the experience of loneliness in romantic and intimate-personal relationships

The study was conducted in early 2020, before the COVID-19 pandemic. The statistical program SPSS21 version was used for data processing, and multidimensional analysis of variance (MANOVA) was applied.

\section{Results}

The analysis of loneliness is a holistic experience for individuals, but the study of this phenomenon requires it to be considered in detail and to analyze its main components "emotional loneliness", "social loneliness" and "romantic/intimate-personal loneliness".

These trends in the intercultural aspect provide an opportunity and are a reliable basis for constructing programs for work on overcoming experiences of loneliness in its detailed version, which will increase the quality of life of people from early, middle and late adulthood as well in Russia and especially in Bulgaria.

Figure 1 shows that statistically, the most significant difference between Russians and Bulgarians was on the scale of "romantic loneliness" $(\mathrm{F}=16.154 ; \mathrm{p} \leq 0.001)$ and then on "emotional loneliness" $(\mathrm{F}=14.266 ; \mathrm{p} \leq 0.001)$. No statistically significant differences were found on the "social loneliness" scale.

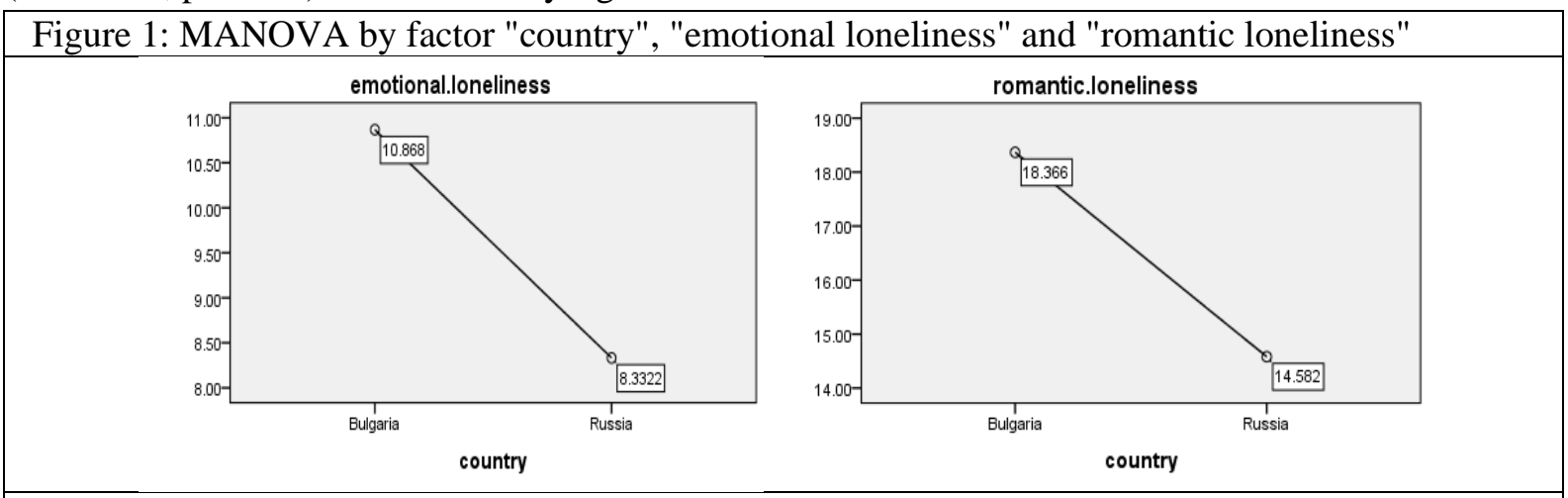

Source: Authors

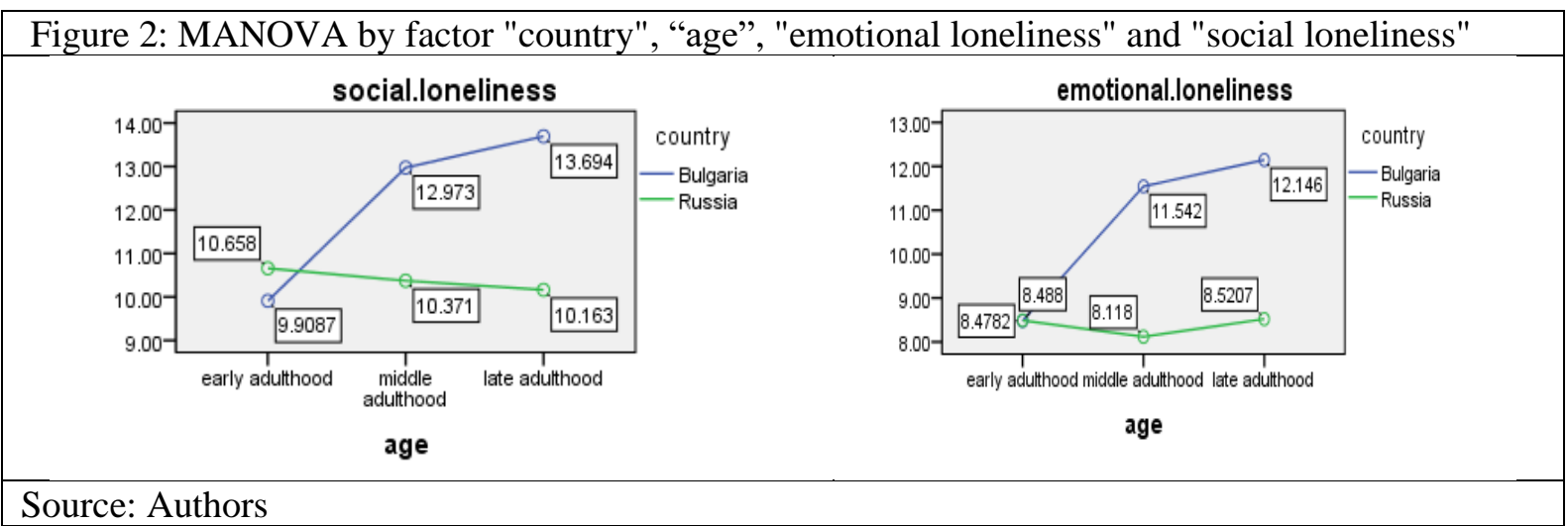

Figure 2 shows that statistically, the most significant difference between Russians and Bulgarians was on the scale of "social loneliness" $(\mathrm{F}=5.245 ; \mathrm{p}=0.006)$ and then on "emotional loneliness" $(\mathrm{F}=3.058$; $\mathrm{p}=0.048$ ). No statistically significant differences were found on the "romantic loneliness" scale.

Figure 3 and Table 1 shows that statistically, the most significant difference was by factor "marital status" between Russians and Bulgarians on the scale of "romantic loneliness" (F=9.351; $\mathrm{p} \leq 0.001)$ and 
also on "emotional loneliness" ( $\mathrm{F}=3.782 ; \mathrm{p}=0.005)$. No statistically significant differences were found on the "social loneliness" scale.

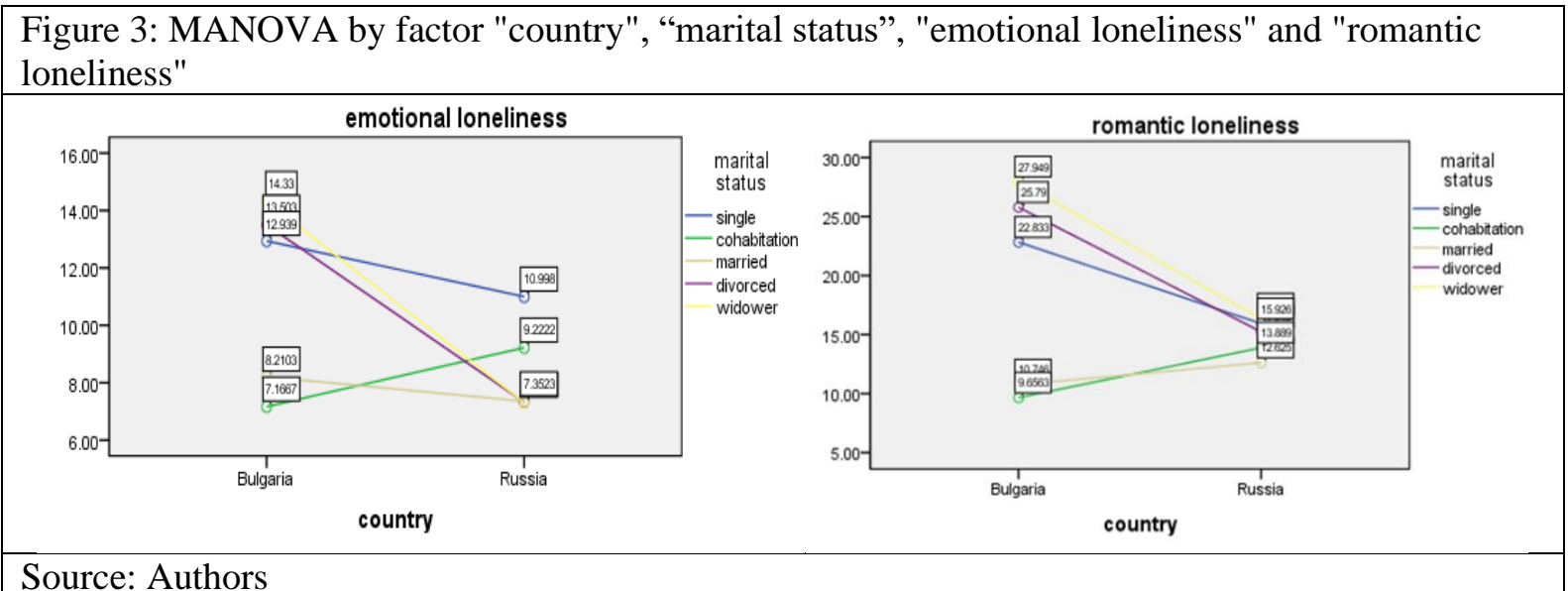

\begin{tabular}{|c|c|c|c|c|}
\hline \multirow[t]{2}{*}{ Marital status } & \multicolumn{2}{|c|}{ Emotional loneliness } & \multicolumn{2}{|c|}{ Romantic loneliness } \\
\hline & Bulgaria & Russia & Bulgaria & Russia \\
\hline Single & 12.939 & 10.998 & 22.833 & 15.232 \\
\hline Cohabitation & 7.1667 & 9.2222 & 9.6563 & 12.625 \\
\hline Married & 8.2103 & 7.3523 & 10.746 & 13.889 \\
\hline Divorced & 13.503 & 7.3063 & 25.79 & 15.926 \\
\hline Widower & 14.33 & 7.3219 & 27.949 & 16.342 \\
\hline
\end{tabular}

In general, the sample of respondents from Russia is more complete, almost homogeneous in the frequency of experiences "emotional loneliness" in relation to divorced, widowed, and married persons. The group of unmarried and those living in cohabitation as a group with a higher frequency of experiencing deficits within the framework of emotions and relationships in the family is clearly distinguished.

In this line of thought, the probability of serious psychological work is needed primarily by the abovementioned groups in Bulgaria, while those in need of such intervention are primarily subgroups of single people or cohabitants from Russia.

A partial explanation of this feature should be sought by focusing on the presented analyzes of the results related to the general presentation and analysis of this subtype of loneliness in the two countries studied.

\section{Discussion}

The meaningful presentation of the subtype "romantic loneliness" is most clearly present in the threecomponent structure of the psychological phenomenon - the experience of loneliness. The characteristics of this subtype are intertwined with deficits in the feeling of lack or presence of personal satisfaction and expectations in the intimate, deeply emotional life of the subjects. We assume that the moderately expressed power distance, the manifestation of collectivism, and the moderately feminine dimension, defining the Bulgarian culture, do not guarantee, but are not a socio-psychological condition for the manifestation of serious, significant, and significant dimensions negative experiences for the studied Bulgarian persons. Manifestation of this subtype of loneliness shows that there is something to be desired to improve the quality of life of the studied elderly people in Bulgaria in the direction of personal support and assistance (Minkov, 2007).

In principle, the tendency for a strong frequency of manifestation of the subtype "romantic loneliness" is common, both for Bulgaria and Russia. In this regard, we can emphasize that there is a general tendency to observe a significant predominance of deficits, shortages, and expectations for the respondents from Bulgaria. Here again, we must point out that the observed differences have their explanation through the prism of the cross-cultural features of the two cultures and the different volumes of the studied samples. 
It became clear that the manifestations of "emotional loneliness" were more pronounced in the subjects from Bulgaria / 10.868/, then in the studied sample from Russia / 8. 3322 /. The interpretation of the observed trends can be viewed through the prism of the already established, as mentioned above, crosscultural differences. These are differences based on and focused on the family's value system, school, living environment. Above all, we could look for an explanation for the strong degree of high preference for avoiding uncertainty, which means that Russian respondents' family and family relationships are supported by rules and beliefs, and at the same time, innovations can meet with resistance. In this sense, security in the family is an important stimulus for individual motivation, for subjective well-being (Sergeeva, 2017). All these features and characteristics have been established with the help of Hert Hofstede's model in the framework of extensive multicultural research. In this regard, the Bulgarian sample and Russia are also defined by similar characteristics, but the manifestation of this dimension is expressed in lower values, which defines it as more moderately avoiding uncertainty with moderate acceptance of risks in family relationships (The Hofstede center. National cultures. Countries).

Moreover, the strong power distance within the Russian sample does not mean a strong "emotional loneliness", while the less pronounced dimension "power distance" in the Bulgarian sample represents a stronger manifestation of this subtype of loneliness, i.e., observed inversely proportional to the values for power distance and the frequency of manifestation of "emotional loneliness". In this sense, the moderate power distance correlates with a strong subcomponent "emotional loneliness" in the respondents from Bulgaria. While the strong power distance among those studied by Russia characterizes them with a lower frequency of manifestations of "emotional loneliness". Analyzing the manifestation of "emotional loneliness" is one of the ingredients of the experience of loneliness.

The analysis of the manifestation of "emotional loneliness" as one of the ingredients of the experience of loneliness during different stages of adulthood in Bulgaria and Russia is generalized in its entirety and presents a more general trend of stronger expression in average marginal values in age subgroups. Middle and late adulthood. In early adulthood, the experience of "emotional loneliness" is similar in value for the two samples - Bulgaria and Russia.

As we have already noted, this once again points to the need to update research on cross-cultural differences. On the other hand, the strong "emotional loneliness", especially in late and middle adulthood subjects, have their explanatory interpretation through the prism of the established intercultural differences in the context of the theory and research of $\mathrm{H}$. Hofstede and the dimensions derived from it.

We cannot but emphasize that there is a relative homogeneity of the frequency of manifestation of this subcomponent of loneliness in relation to the average marginal values for the studied persons in early, middle, and late adulthood. The data indicate the need for psychological support and prevention for the surveyed persons in middle and late adulthood in Bulgaria.

When we looked at the results on the scale "emotional loneliness" for Bulgarians and Russians by factor "marital status", we found that, the results give us reason to define this subtype of loneliness as the most convincingly expressed among the unmarried, followed by divorced and widowed widows in Bulgaria. Similar trends were observed in the sub-sample of singles in Bulgaria /12.939/ and Russia /10.998/. But here, too, there is a tendency for emotional loneliness to be more differentiated among Bulgarians in terms of „marital status“. In the severity of this trend, we can assume that there are unrealized expectations and attitudes for home and family, personal and insurmountable bitterness, unsuccessful life situations, events and more. The "emotional loneliness" is relatively stronger in the sample from Russia in the status of "cohabitation".

The present study also has some limitations. Although the sample is representative, there is a lack of gender homogeneity - only $36 \%$ of male subjects were surveyed. This is mainly due to the tendency in both Russia and Bulgaria for women to respond more often to invitations to complete surveys. The gender ratio is a significant limitation in the study, and therefore we cannot be sure of the interpretation of gender data. Also, it would be good to confirm these results with another methodology for measuring loneliness because in this way, their validity will be proven.

\section{Conclusion}

From the presented three-component model for the study of loneliness, our results outline the "romantic and intimate-personal loneliness" as the most clearly manifested and present with its frequency in the content of the overall experience of loneliness. This outlines deficits, expectations, and emotions, which 
are realized in the deeply personal world of the person, of the studied groups of adults both in Bulgaria and in Russia. There is a strong tendency to experience romantic / intimate-personal loneliness among Bulgarians

In second place in terms of its representation is the "emotional loneliness", which stands out among the studied adults from Bulgaria according to its marginal values. We need to point out that here, too, although there are parallel trends for both Bulgaria and Russia, but in terms of frequency of occurrence, this subtype of loneliness is more characteristic of the Bulgarian sample. In our opinion, part of the explanation is in the different sample sizes, but the explanation is also similar in content experiences and deficits in the relationships in the circle of friends.

Loneliness often occurs for reasons beyond human control. In such cases, it arises as a result of a sudden complete or partial emotional and psychological isolation of the person from the people who were the main circle of communication. In the pursuit of the best adaptation to the environment, it is assumed that a person creates connections and contacts to which he is attached to some extent. Deficiency in any of these different types of relationships can lead to either emotional or social loneliness. It should be noted that people perceive loneliness differently. Some do not experience loneliness at all or experience it to a small degree. Usually, these are people who have felt the need for loneliness in earlier stages of their lives.

\section{Acknowledgments}

The Bulgarian part of the article was supported by the project of Bulgarian Research Fund, Project No. KP-06-Russia14, entitled "Loneliness vs. independence across the lifespan: Perspectives and insights from Bulgaria and Russia".

The Russian part of the article is implemented with the financial support of the Russian Fund for Basic Research (RFBR) in the framework of a research project № 19-513-18015

\section{References}

Alexandrova N. (2015). A little book about the great loneliness. Sofia, Bulgaria: Prof. Petko Venedikov, 129 p. (in Bulgarian)

Alexandrova, N., Babakova, L., Velkov, Yu. (2020). Influence of economic factors on the experience of loneliness in adulthood. In Sat. "Personality, well-being and health in the organization", pp.696-703 (in Bulgarian)

Di Tommaso E., Brannen C., Best L.A.(2004). Measurement and validity characteristics of the Short Version of the Social and Emotional Loneliness Scale for Adults // Educational \& Psychological Measurement. Vol. 64 (1). P. 99—119. DOI: $10.1177 / 0013164403258450$

DiTomasso, E. i Spinner, B. (1993). The development and initial validation of the social and emotional loneliness scale for adults (SELSA). Personality and Individual Differences, 14, 127-134.

Junttila, N., Ahlqvist-Björkroth, S., Aromaa, M., Rautava, P., Piha, J.,Vauras, M., et al. (2013). Mothers' and fathers' loneliness during pregnancy, infancy, and toddlerhood. Psychology and Education, 50, 98-104

Lacković-Grgin K. (2008). Usamljenost: Fenomenologija, teorije i istraživanja. Jastrebarsko: Naklada Slap

Minkov, M. (2007). Why we are different. Sofia: Klasika I stil, pp. 264, ISBN 9789543270293 (in Bulgarian)

Osin E.N., Leontiev, D.A. (2013). Differential questionnaire of loneliness experience: structure and properties. In Psychology. Journal of the Higher School of Economics, 2013. T. 10, No. 1. P. 55-81. (in Russian)

Russell, D. (1982). The measurement of loneliness. U: L. A. Peplau i D. Perlman (Eds.), Loneliness: A sourcebook of current theory, research, and therapy (pp. 81-104). New York, NY: Wiley.

Safonova, M.V. (2012). Social ideas of modern Russians about the lonely person. In "Problems of psychology in the 21 st century", Volume 4, pp. 71-84, ISSN 2029-8587 (in Russian)

Sergeeva, A.V. (2017). Russians. Behavior stereotypes, traditions, mentality. Flinta-Nauka, pp. 440, ISBN: 978-5-89349626-0 (in Russian).

Strizhitskaya O. Yu., Murtazina IR, Babakova LV, Alexandrova N. Kh. (2020). Representations of loneliness in Russia and Bulgaria (on the example of students) // Izv. Sarat. un-ta. New sir. Ser. Acmeology of education. Developmental psychology. T. 9, issue. 4 (36). Pp. 367-376. DOI: https://doi.org/10.18500/2304-9790-2020-9-4-367-376 (in Russian)

Strizhitskaya OY, Petrash MD, Murtazina IR, Vartanyan GA, Manevsky FS, Alexandrova NH, Babakova LV (2020). Adaptation of the Bulgarian version of the scale of social and emotional loneliness (SELSA-S) for adults and the elderly // Consultative Psychology and Psychotherapy. Volume 28. № 4. pp. 79-97. doi: 10.17759 / cpp.2020280405 (in Russian)

The Hofstede centre. National cultures. Countries (n.d.). Retrieved from: http; //geert-hofstede.com/

Vanhalst, J., Luyckx, K. \& Goossens, L. (2014). Experiencing loneliness in adolescence: A matter of individual characteristics, negative peer experiences, or both? Review of Social Development, 23, 100-118.

Weiss, R. S. (1973). Loneliness: The experience of emotional and social isolation. Cambridge, MA: MIT Press.

Zhao, J., Song, F., Chen, Q., Li, M. \& Kong, F. (2018). Linking shyness to loneliness in Chinese adolescents: The mediating role of core self-evaluation and social support. Personality and Individual Differences, 125, 140-144. 